\title{
Study on the Traffic Energy System Model in Urumqi Based on Scenario Analysis Methods
}

\author{
Xiaowei Sun ${ }^{1}$, Yulei Xie ${ }^{1}$, Zhenghui Fu ${ }^{1}$, Hongkuan Zang ${ }^{2}$ \\ ${ }^{1}$ North China Electric Power University, Key Laboratory of Regional Energy System Optimization, \\ Ministry of Education, Beijing, China \\ ${ }^{2}$ Chinese Academy for Environmental Planning, Beijing, China \\ Email: xiaoweinumberone@163.com,weili819@yahoo.com.cn
}

Received December, 2012

\begin{abstract}
In this study, a traffic energy system model is developed to optimize the traffic system cost of Urumqi, considering energy consumption, pollution emission and travel time. Meanwhile, scenario analysis method is proposed to solve the problem of the extreme weather of traffic, and three scenarios (i.e. $10 \%, 20 \%$ and $30 \%$ ) of reductions of traffic flow quantity and pollutant emission are examined. The results demonstrate that the medium-type coach will be the promising selection under different scenarios especially in the extreme conditions and the traffic flow reduction scenarios are not the better option for the decision owing to the same cost under the different reduction levels. Moreover, encouraging the medium-type coach traveling and restricting the small vehicle driving would be attractive alternatives for the extreme situation. The proposed model would provide reasonable references for decision makers.
\end{abstract}

Keywords: Traffic Energy System; Urumqi; Scenario Analysis; Pollutant Emission; Traffic Flow Quantity

\section{Introduction}

The number of vehicles has increased rapidly with the progress of urbanization. It is reported that the motor vehicle quantity has increased from 219 million in August in 2011 to 233 million in June in 2012. Moreover, the fuel demand is predicted to 230 and 370 million tons in 2020 and 2030 respectively, which will take the proportion of $57 \%$ and $70 \%$ of the year. In addition, the great increase of travel demands in urban area brings negative effect on environment. According to the data of World Bank in 1997, though the vehicle quantity of Tokyo was ten times of Beijing, they had the same pollutant emission which demonstrated the significant importance of proper traffic mode to reducing the pollutant emission and the capital cost. The deterioration of air quality is one of the most pressing environmental concerns today due to vehicle emissions (e.g. $\mathrm{NO}_{\mathrm{x}}, \mathrm{CO}$ ). For example, the vehicular emission of $\mathrm{CO}, \mathrm{HC}$ and $\mathrm{NO}_{\mathrm{x}}$ took up $63 \%$, $73 \%$ and $28 \%$ of the whole emission in Beijing and $86 \%$, $90 \%$ and $56 \%$ in Shanghai[1]. Urban air pollution influences not only the health of citizens but also the sustainable development of cities.

In the past decades, many researchers devoted themselves to the traffic environmental analysis and developed a number of air traffic environment impact assessment models. For example, Benson proposed a dispersion model for predicting air pollutant concentrations[2].
$\mathrm{Fu}$ et al. used the MOBILE5 model to calculate the composite emission factors of the vehicle fleet in Beijing and achieved the overall emission amounts and the contribution rate of automobiles for the urban areas as well as Beijing[3]. Zi et al. investigated the vehicle driving patterns on different roads in Ningbo city using advanced GPS technique, analyzed the situation of motor vehicle emission pollution and its influence factors and emphatically discussed the effects of vehicle models and the driving cycle on vehicle emission pollutants[4]. Zhao discussed the atmospheric environmental carrying capacity of urban road traffic from the macro level and meso level respectively, and proposed the mathematical model of traffic atmospheric environmental carrying capacity to forecast the maximal owned quantity of motor vehicle within the urban area and constructed the model describing mathematical relationship between emission intensity and traffic flow[5].

However, most of them focused on the vehicle environmental models, and few studies took extreme weather into consideration to be combined with the scenario analysis methods[6-8]. Weather takes an important role in transportation, and different vehicles have different contributions on the air pollution of vehicle exhaust emission. Thus, a traffic energy model is developed based on different types of vehicles, environmental capacity, energy consumption and traveling time. It would 
provide a scientific basis for the development of traffic energy system of Urumqi to reduce vehicle emissions and, therefore, to reach its environmental objectives. The optimum vehicles distribution of transportation energy system would be obtained, which are valuable for decision makers to identify desired vehicle ban policy.

\section{Case Study}

\subsection{Overview of the Study Area}

Urumqi, capital of Xinjiang Uygur Autonomous Region,

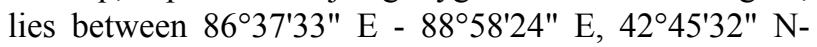
$44^{\circ} 08^{\prime} 00^{\prime \prime} \mathrm{N}$, situated on the northern piedmont plains (about $800 \mathrm{~m}$ above sea level) of the Tianshan Mountains[9]. It is located in temperate continental climate zone and the average temperature throughout of a year is about $6.5^{\circ} \mathrm{C}$. Temperature inversion appears in the whole year, particularly in winter. Owing to the adverse geographic conditions, unreasonable energy structure, intensive urban layout and undeveloped environmental management, the air quality of Urumqi can hardly meet the secondary standard of national ambient quality. Meanwhile, it has a population of over 3.11 million in 2010, increased by $49.51 \%$ than 2000 , which means a more travelling and fuel demands. What's worse, the vehicle quantity has reached to 0.46 million in 2012 almost three times than the number in 2006. In recent years, environmental deteriorations have been increasingly prominent due to rapid population growth and speedy socio-economic development (e.g. vehicles and manufactures). Moreover, the environmental impacts of vehicles are more serious in the extreme weather to the traffic energy system. Therefore, it's desired to conduct efforts to ameliorate the current situation of air environment.

\subsection{Model Formulation}

As the lifeline of transportation development, the urban traffic energy system is always associated with complicated factors, such as energy consumption types, travel modes and transport volume. In this study, a three-year planning horizon is selected. Four districts (e.g. Tianshan, Shuimogou, Saybagh and High-tech districts) and several main roads (e.g. Xihong Road, Nanhu Road, Altay Road, Northwest Road, Karamay West Street, Beach Expressway and the out ring road) are considered, as shown in Figure 1.

The objective of the model is to minimize transportation system consumption, in view of the serious consumption of traffic resource, the lack of non-renewable resource and the unbalanced use for resource supply. Meanwhile, the environmental load cost, the travelling time cost and the traffic energy consumption cost are taken into consideration simultaneously. In addition, a

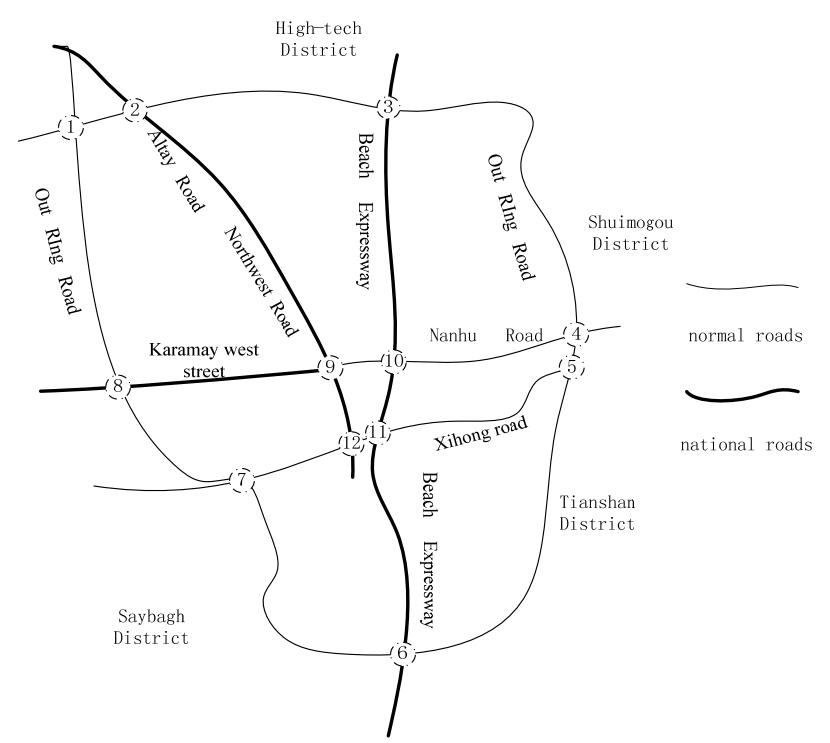

Figure 1. Schematic diagram of the study area.

number of constraints (e.g. road volume limitation, pollutant emission standard) are examined, such as road volume limitations, citizen's travel demands and vehicle pollutant emissions. Thus, the urban traffic energy system optimization model can be formulated as follows:

$$
\operatorname{Min} f=\mathrm{C}_{\mathrm{EL}}+\mathrm{C}_{T T}+\mathrm{C}_{E \mathrm{C}}
$$

a) Environmental load cost:

$$
\mathrm{C}_{E L}=\sum_{s=1}^{3} \sum_{k=1}^{3} \sum_{i=1}^{I} \sum_{j=1}^{J} \sum_{v t=1}^{3} \alpha_{k}\left(\mathrm{RL}_{s, i, j} \cdot \mathrm{m}_{s, k, i, j, v t} \cdot \mathrm{VS}_{s, i, j, v t}\right)
$$

where $i$ represents original node of the roads; $j$ denotes destination node of the roads; $k$ means the pollutants, where $k=1$ for $\mathrm{CO}$ (Carbonic Oxide), $k=2$ for THC (Total Hydrocarbon Compounds) and $k=3$ for $\mathrm{NO}_{2}$ (nitrogen dioxide); $s$ is the planning horizon, $k=1,2,3$; $v t$ is the vehicle types, $v t=1$ for the medium-type coach, $v t=$ 2 for the small vehicle and $v t=3$ for the large truck; $\alpha_{k}$ is the loss index of unit pollutants, and $\alpha_{k=1}=11.62$ $\$ / \mathrm{kg}, \quad \alpha_{k=2}=968.75 \$ / \mathrm{kg}, \quad \alpha_{k=3}=387.5 \$ / \mathrm{kg}[10]$; $\mathrm{RL}_{s, i, j}$ denotes the length of the road with original node $i$ and destination node $j$ at period $s(\mathrm{~km})$ (showed in Table 2); $\mathrm{m}_{s, k, i, j, v t}$ is the vehicle emission coefficient of different pollutants $k$ with the mean speed of the road with original node $i$ and destination node $j$ at period $s$ (the values can be calculated in interpolation algorithms combined with the traffic flow characteristics, showed in Table 1)[11]; $\mathrm{VS}_{s, i, j, v t}$ is the traffic volume of vehicle type $v t$ on the road with original node $i$ and destination node $j$ at period $s$ (vehicle/min).

b) Traveling time cost

$$
\mathrm{C}_{T T}=\mathrm{C}_{0} \cdot \sum_{s=1}^{3} \sum_{i=1}^{I} \sum_{j=1}^{J} \mathrm{t}_{s, i, j, v t} \cdot \mathrm{VS}_{s, i, j, v t}
$$


Table 1. Vehicle exhaust emission coefficients.

\begin{tabular}{|c|c|c|c|c|c|c|c|}
\hline \multirow{2}{*}{ Vehicle Types } & \multirow{2}{*}{ Pollutants } & \multicolumn{6}{|c|}{ Average speed $(\mathrm{km} / \mathrm{h})$} \\
\hline & & 50 & 60 & 70 & 80 & 90 & 100 \\
\hline \multirow{3}{*}{ Small Vehicle } & $\mathrm{CO}$ & 31.34 & 23.68 & 17.90 & 14.76 & 10.24 & 7.72 \\
\hline & $\mathrm{THC}$ & 8.14 & 6.70 & 6.06 & 5.30 & 4.66 & 4.02 \\
\hline & $\mathrm{NO}_{\mathrm{x}}$ & 1.77 & 2.37 & 2.96 & 3.71 & 3.85 & 3.99 \\
\hline \multirow{3}{*}{ Medium-type Coach } & $\mathrm{CO}$ & 30.18 & 26.19 & 24.76 & 25.47 & 28.55 & 34.78 \\
\hline & $\mathrm{THC}$ & 45.21 & 12.42 & 11.02 & 10.10 & 9.42 & 9.10 \\
\hline & $\mathrm{NO}_{\mathrm{x}}$ & 5.40 & 6.30 & 7.20 & 8.30 & 8.80 & 9.30 \\
\hline \multirow{3}{*}{ Large Truck } & $\mathrm{CO}$ & 5.25 & 4.48 & 4.10 & 4.01 & 4.23 & 4.77 \\
\hline & $\mathrm{THC}$ & 2.08 & 1.79 & 1.58 & 1.45 & 1.38 & 1.35 \\
\hline & $\mathrm{NO}_{\mathrm{x}}$ & 10.44 & 10.48 & 11.10 & 14.71 & 15.64 & 18.38 \\
\hline
\end{tabular}

Table 2. Length of different roads.

\begin{tabular}{cccccc}
\hline Road & Length $(\mathrm{km})$ & Road & Length $(\mathrm{km})$ & Road & Length $(\mathrm{km})$ \\
\hline $\mathrm{r}_{1,2}$ & 1.54 & $\mathrm{r}_{7,8}$ & 2.53 & $\mathrm{r}_{3,10}$ & 3.76 \\
$\mathrm{r}_{2,3}$ & 3.57 & $\mathrm{r}_{8,9}$ & 1.97 & $\mathrm{r}_{4,10}$ & 2.97 \\
$\mathrm{r}_{3,4}$ & 5.43 & $\mathrm{r}_{9,10}$ & 1.36 & $\mathrm{r}_{5,11}$ & 3.44 \\
$\mathrm{r}_{4,5}$ & 0.55 & $\mathrm{r}_{10,11}$ & 1.51 & $\mathrm{r}_{6,11}$ & 3.80 \\
$\mathrm{r}_{5,6}$ & 6.62 & $\mathrm{r}_{11,12}$ & 0.54 & $\mathrm{r}_{7,12}$ & 1.50 \\
$\mathrm{r}_{6,7}$ & 4.28 & $\mathrm{r}_{2,9}$ & 4.29 & $\mathrm{r}_{9,12}$ & 1.76 \\
\hline
\end{tabular}

where $\mathrm{t}_{s, i, j, v t}$ is the travel time of vehicle type $v t$ on the road with original node $i$ and destination node $j$ at period $s(\mathrm{~min}) ; \mathrm{C}_{0}$ is the coefficient of time value (\$).

c) Energy consumption cost

$$
\mathrm{C}_{E C}=\sum_{s=1}^{3} \sum_{i=1}^{I} \sum_{j=1}^{J} \sum_{v t=1}^{3} \mathrm{FU}_{s} \cdot \mathrm{VS}_{s, i, j, v t}\left(\mathrm{FB}_{s-1, v t} \cdot \mathrm{RL}_{s, i, j}+\mathrm{FS}_{s, v t}\right)
$$

where $\mathrm{FU}_{s}$ is the fuel price at period $\mathrm{s}(\$ / \mathrm{L}) ; \mathrm{FB}_{s, v t}$ is the mean fuel volume of vehicle type $v t$ at period $s$ $(\mathrm{L} / \mathrm{km}) ; \mathrm{FS}_{s, v t}$ is the mean fuel volume of vehicle type $v t$ under idling at period $s(\mathrm{~L})$.

The model constraints involve relationships among transportation development and related environmental restrictions, which can be categorized into the following three groups.

1) Constraints of road capacity constraints:

$$
\sum_{v t=1}^{3} \mathrm{VS}_{s, i, j, v t} \leq \mathrm{VT}_{s, i, j} \quad \forall s, i, j
$$

where $\mathrm{VT}_{s, i, j}$ is the top vehicle capacity of the road with original node $i$ and destination node $j$ at period $s$ (vehicles).

2) Constraints for traveling demands:

$$
\mathrm{VS}_{\mathrm{s}, i, j, v t} \geq \mathrm{SQ}_{s, i, j, v t} \quad \forall s, i, j, v t
$$

$$
\sum_{v t=1}^{3} \mathrm{VS}_{s, i, j, v t} \geq \mathrm{Q}_{s, i, j} \quad \forall s, i, j
$$

where $\mathrm{SQ}_{s, i, j, v t}$ is the demanding vehicle volume of vehicle type $v t$ on the road with original node $i$ and destination node $j$ at period $s$ (vehicles/min); $\mathrm{Q}_{\mathrm{s}, \mathrm{i}, \mathrm{j}}$ represents the demanding vehicle flow quantity of the road with original node $i$ and destination node $j$ at period $s$ (vehicles/min).

3) Constraints for pollutants emission:

$$
\sum_{i=1}^{\mathrm{I}} \sum_{j=1}^{J} \sum_{v t=1}^{3}\left(\mathrm{RL}_{s, i, j} \cdot \mathrm{m}_{\mathrm{s}, k, i, j, v t} \cdot \mathrm{VS}_{s, k, i, j, v t}\right) \leq \mathrm{UPE}_{s, k} \quad \forall s, k
$$

$$
\sum_{v t=1}^{3}\left(\mathrm{RL}_{s, i, j} \cdot \mathrm{m}_{\mathrm{s}, k, i, j, v t} \cdot \mathrm{VS}_{s, k, i, j, v t}\right) \leq \mathrm{UPER}_{s, k, i, j} \quad \forall s, k, i, j
$$

where $\mathrm{UPE}_{\mathrm{s}, \mathrm{k}}$ is the emission concentration constraints of the traffic system for pollutant $k$ at period $s$ (tons/year); $\mathrm{UPER}_{\mathrm{s}, \mathrm{k}, \mathrm{i}, \mathrm{j}}$ is the emission concentration constraints of the road with original node $i$ and destination node $j$ at period $s$ (tons/year).

\section{Results and Discussion}

Owing to the typical temperate continental climate and special weather conditions, the fog haze weather happens frequently and takes a great impact on the traffic energy system of Urumqi. Thus, it is an urgent to develop a traffic energy optimization model based on scenario analysis methods considering the reductions of the traffic flow and the pollutant emission quantity. The vehicle flow quantity of the main roads under different scenarios can be obtained, which are valuable for decision makers in the management practices. 


\subsection{Scenario Analysis of the Traffic Flow}

Motor vehicle emissions contribute significantly to air pollution in urban areas, and main pollutants include carbon monoxide $(\mathrm{CO})$, hydrocarbons $(\mathrm{HC})$, sulfur oxides $\left(\mathrm{SO}_{\mathrm{x}}\right)$, and photochemical oxidants. What's more, the pollution emissions depend not only on the vehicle types but also on the traffic flow on the roads. When the extreme weather happens, the decrease of the traffic flow can reduce the impact of the pollutant emissions. Therefore, the developed model aims to optimize the traffic flows of the main roads in Urumqi to meet the environmental and energy demand. Figures $\mathbf{2}$ and $\mathbf{3}$ show the optimized results under scenarios of original level and three different reduction levels (e.g. 10\%, 20\%, 30\%). Though the number of small vehicles will increase by the periods under the original level, the medium-type coaches do the same under the other levels. When the traffic flow remains original level, the traffic flow of $r_{9,12}, r_{3,10}$ and $\mathrm{r}_{6,11}$ would have a big variation both in the vehicle types and flow quantity at different periods. Moreover, in the same decrease level, the vehicle flow remains the same proportion trend at different periods. It is that the medium-type coach would increase obviously at different periods (e.g. the vehicle flow quantity at period 2 showed in Figure 3), especially in $r_{6,7}, r_{7,8}, r_{2,9}, r_{9,12}, r_{3,10}, r_{10,11}$, and $\mathrm{r}_{8,9}$. It is indicated that the medium-type coach would be a better alternative of travel vehicle. In different decrease levels, the vehicle flow of the same vehicle type on different roads would not vary significantly at the same period. However, the same objective costs would be achieved under different decrease levels. It indicates that the decrease of the traffic flow is not a better alternative to the traffic energy system especially in the urgent traveling demands of the city sustainable development.

\subsection{Scenario Analysis of the Pollutant Emission Quantity}

When the extreme weather appears, the vehicle emission should be reduced to some level to avoid the environment impact to the traffic energy system. In this study, three reduction levels of pollutant emissions (i.e. $10 \%$, $20 \%$ and $30 \%$ ) are examined. The system costs under different levels are presented in Table 3. It demonstrates that the traffic system cost would increase initially with the raised reduction levels. Figure 4 shows the traffic flows under the different levels. It is recognized that, the traffic flow differs a little between the $10 \%$ reduction and the normal level of pollutant emissions at different periods (e.g. at period 2). When the reduction levels are $20 \%$ and $30 \%$, the amounts of small vehicles decrease dramatically instead the medium-type coach increases significantly. However, the proportion of the small ones will obtain some increase under the $30 \%$ reduction. Obvious variation of vehicle types and amount appears on $\mathrm{r}_{6,7}, \mathrm{r}_{7,8}$,

(a) at period 1
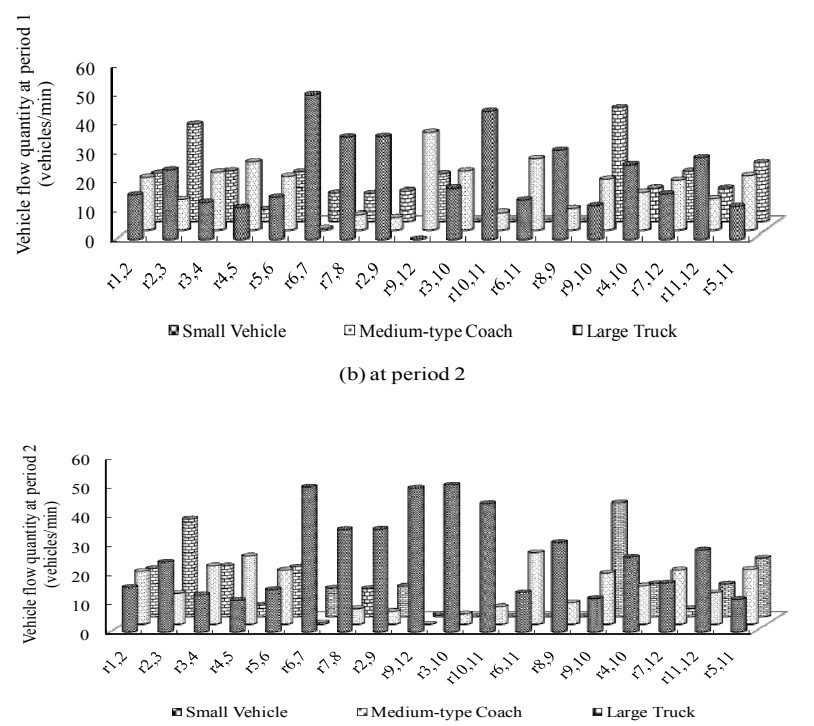

(c) at period 3

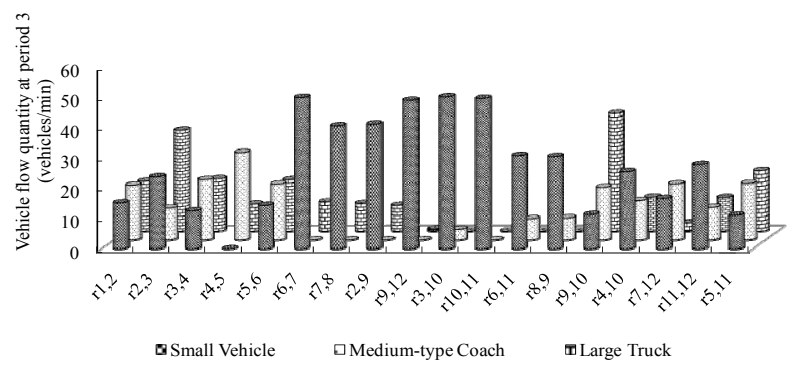

Figure 2. Vehicle flow quantity of different roads at different periods.

(a) Normal traffic flow

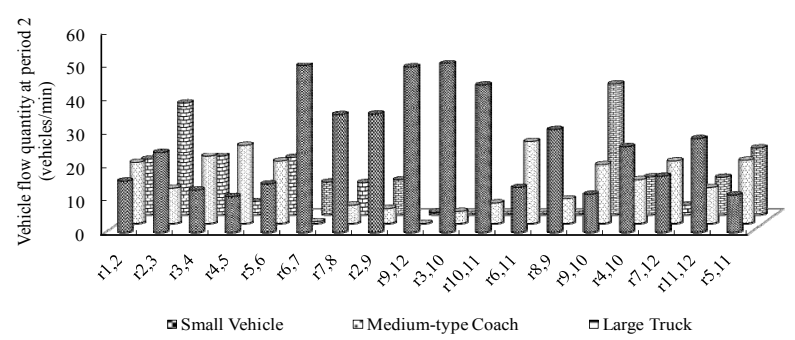

(b) $10 \%$ reduction of the traffic flow

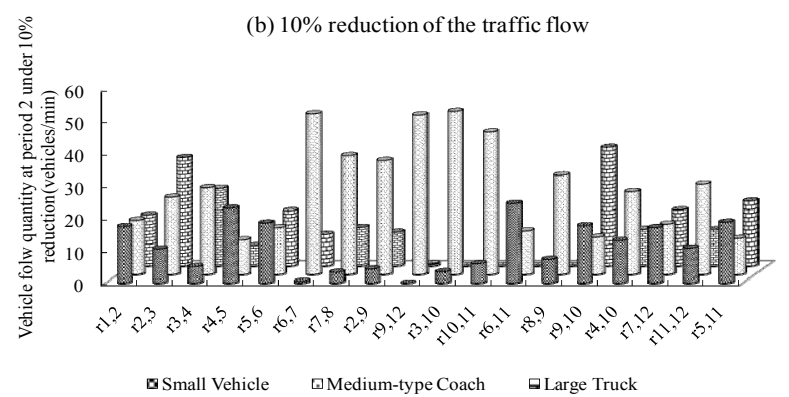



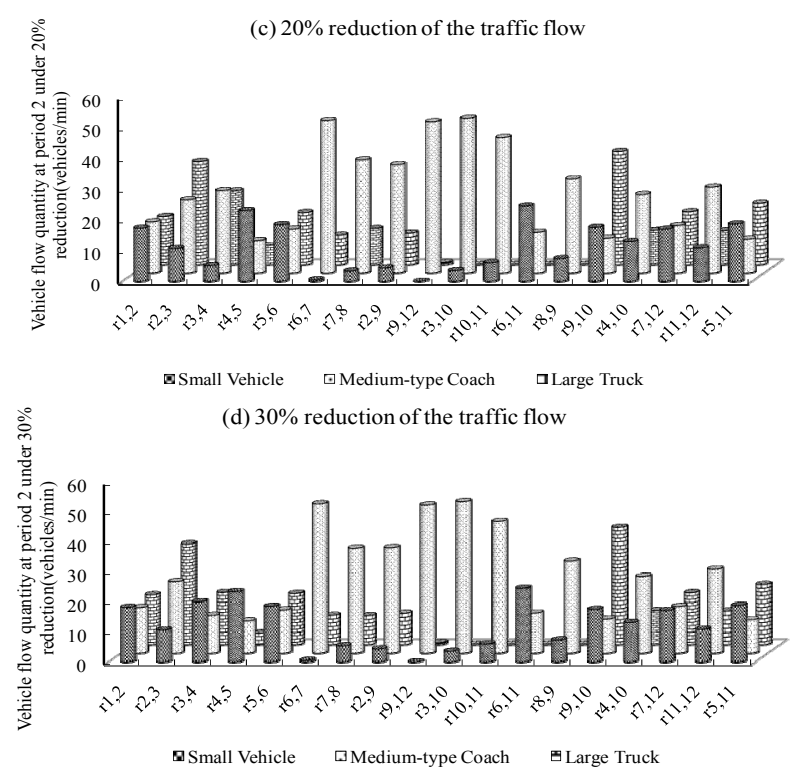

Figure 3. Vehicle flow quantity of different roads at period 2 under different flows.

Table 3. Objective cost of different pollutant emission.

\begin{tabular}{cc}
\hline Scenarios & Objective cost $(\$)$ \\
\hline Original & 92965.10 \\
$10 \%$ of reduction & 94683.68 \\
$20 \%$ of reduction & 99225.93 \\
$30 \%$ of reduction & 100482.20 \\
\hline
\end{tabular}

(a) Normal pollutant emissions

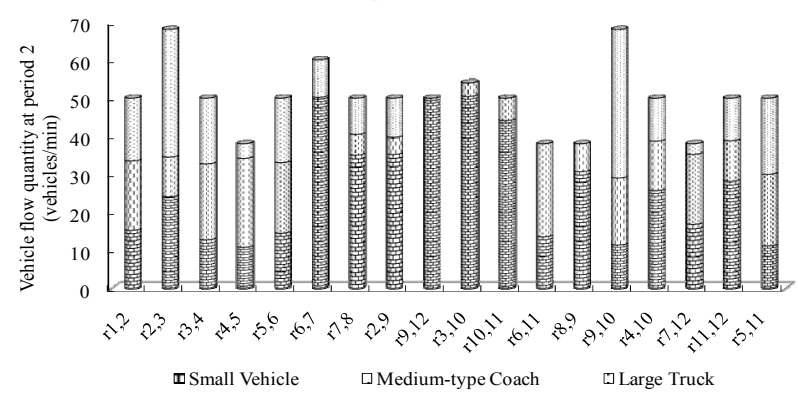

(b) $10 \%$ reduction of the pollutant emissions

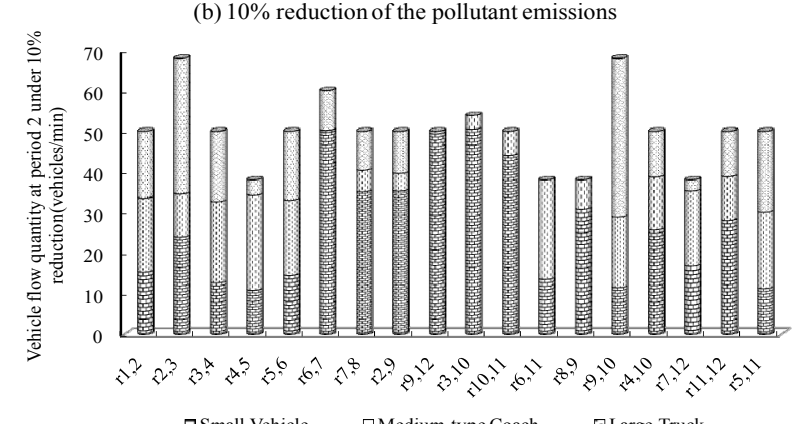

(c) $20 \%$ reduction of the pollutant emissions

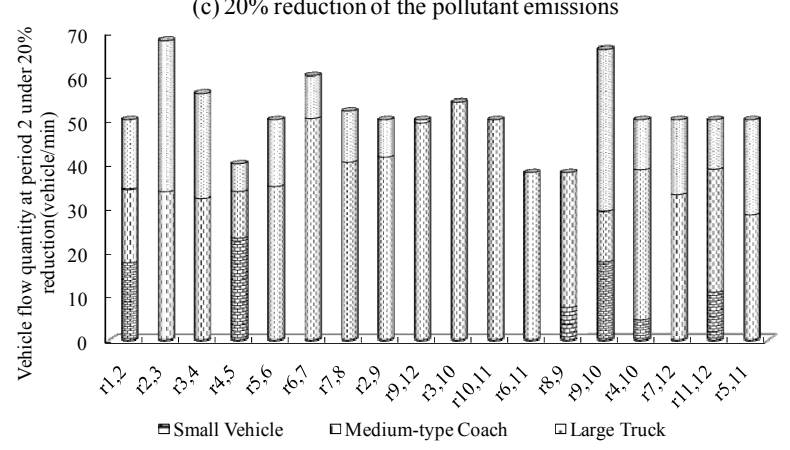

(d) $30 \%$ reduction of the pollution emissions

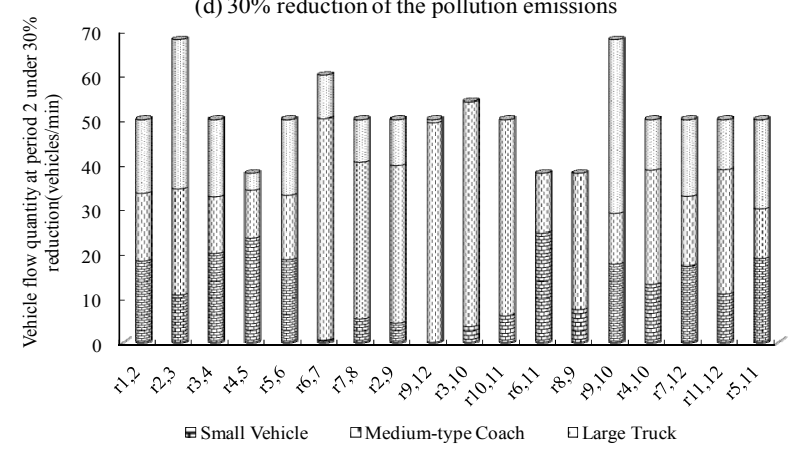

Figure 4. Vehicle flow quantity of different roads at period 2 under different pollutant emissions.

$\mathrm{r}_{2,9}, \mathrm{r}_{9,12}, \mathrm{r}_{3,10}, \mathrm{r}_{10,11}, \mathrm{r}_{8,9}$ and $\mathrm{r}_{6,11}$. In addition, the amount of the large truck should always keep a low level. This indicates that small vehicles play an important role in the pollution emissions. In the long run, the medium-type coach and the large truck would have the increasing trends and take large proportions. It also indicates that the citizen should take the medium-type coaches not only to protect the environment but also to satisfy the traveling demands of local citizens which will also a constructional suggestion to the decision-makers.

\section{Conclusions}

A traffic energy system model is developed to optimize the traffic flows on main roads of Urumqi, considering energy consumption, pollution emissions and traveling time based on scenario analysis methods to the traffic flow and pollutant-emission quantity. Meanwhile the vehicles are classified into small vehicles, medium-type coaches and large trucks to further analysis the energy and environmental impact of the different vehicles. The optimized vehicle flow on main roads under different scenarios would be obtained and interpreted, which demonstrates that 1) the decrease of the traffic flow would not be a better alternative to the traffic energy system due to the same cost, 2) $r_{6,7}, r_{7,8}, r_{2,9}, r_{9,12}, r_{3,10}, r_{10,11}, r_{8,9}$ and $\mathrm{r}_{6,11}$ should be given priority to owing to its dramatic variation under different scenarios and 3) the mediumtype coach travel would be the optimizing scheme in the 
future. This model could provide valuable references for vehicle restriction policies.

\section{REFERENCES}

[1] C. L. Lou, "Study on the Relationship of Transportation Mode and Energy Consumption and Exhaust Pollution," Beijing Jiaotong University, 2007.

[2] P. E. Benson, "CALINE4-A Dispersion Model for Predicting Air Pollutant Concentrations near Roadways," Federal Highway Administration Report FHWA/CA/TL-84/15, California State Department of Transportation, Sacramento, California (NTIS PB 85 211498/AS), 1984

[3] L. X. Fu, J. M. Hao, D. Q. He, K. B. He and P. Li, "Assessment of Vehicular pollution in China," Journal of the Air and Waste Management Association, Vol. 51, No. 5, 2001, pp. 658-668.

doi:10.1080/10473289.2001.10464300

[4] K. Zi, Y. Q. Huang, X. K. Tu and R. F. Yang, "Research of Driving Cycle and Emission Pollutant of Vehicles in Ningbo City," Chinese Internal Combustion Engine Engineering, Vol. 27, No. 1, 2006, pp. 81-84.

[5] W. J. Zhao, "Research on Atmospheric Environmental
Carrying Capacity of Urban Road Traffic," Chengdu: Southwest Jiaotong University, 2007.

[6] C. K. Chan and X. H. Yao, "Air Pollution in Mega Cities in China," Atmospheric Environment, Vol. 42, No. 1, 2008, pp. 1-42. doi:10.1016/j.atmosenv.2007.09.003

[7] K. B. He, H. Huo and Q. Zhang, "Urban Air Pollution in China: Current Status, Characteristics, and Progress," Annual Review of Energy and the Environment, Vol. 27, No. 3, 2002, pp. 397-431.

doi:10.1146/annurev.energy.27.122001.083421

[8] J. M. Hao, L. X. Fu, K. B. He and Y. Wu, "Pollution Control of Urban Motor Vehicle Emission," Beijing: China Environmental Science Press, 2001.

[9] B. G. Wei, F. Q. Jiang, X. M. Li and S. Y. Mu, "Spatial Distribution and Contamination Assessment of Heavy Metals in Urban Road Dusts from Urumqi," NW China Microchemical Journal, Vol. 93, 2009, pp. 147-152.

doi:10.1016/j.microc.2009.06.001

[10] H. Nakamura, "Social Economic Evaluation of Road Investment," Journal of Public Economics, Vol. 69,1998, pp. 103-121.

[11] J. Duo, "Study on Diffusion of Motor Vehicle Emission in the Time and Space Based on GIS on the Youhao Road," Urumqi Urumqi: Xinjing University, 2006. 